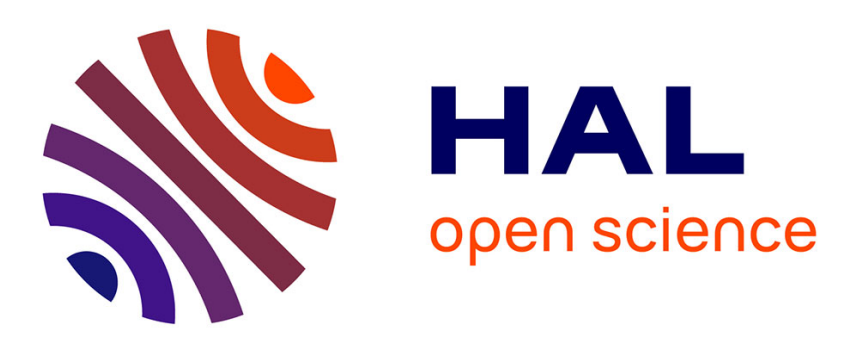

\title{
Large Time-Step Numerical Scheme for the Seven-Equation Model of Compressible Two-Phase Flows
}

Christophe Chalons, Frédéric Coquel, Samuel Kokh, Nicole Spillane

\section{To cite this version:}

Christophe Chalons, Frédéric Coquel, Samuel Kokh, Nicole Spillane. Large Time-Step Numerical Scheme for the Seven-Equation Model of Compressible Two-Phase Flows. Finite Volumes for Complex Applications VI, 2011, Czech Republic. pp. 225-233, 10.1007/978-3-642-20671-9_24 . hal-01018835

\section{HAL Id: hal-01018835 \\ https://hal.science/hal-01018835}

Submitted on 5 Jul 2014

HAL is a multi-disciplinary open access archive for the deposit and dissemination of scientific research documents, whether they are published or not. The documents may come from teaching and research institutions in France or abroad, or from public or private research centers.
L'archive ouverte pluridisciplinaire HAL, est destinée au dépôt et à la diffusion de documents scientifiques de niveau recherche, publiés ou non, émanant des établissements d'enseignement et de recherche français ou étrangers, des laboratoires publics ou privés. 


\title{
Large Time-Step Numerical Scheme for the Seven-Equation Model of Compressible Two-Phase Flows
}

\author{
Christophe Chalons ${ }^{* 1,3}$, Frédéric Coquel ${ }^{\dagger 2}$, Samuel Kokh ${ }^{\ddagger 1}$, and Nicole Spillane ${ }^{\S 3}$ \\ ${ }^{1}$ CEA-Saclay, 91191 Gif-sur-Yvette, France \\ ${ }^{2}$ Ecole Polytechnique, Route de Saclay, 91128 Palaiseau Cedex, France. \\ ${ }^{3}$ Laboratoire J.-L. Lions, UPMC Univ Paris 06, BC 187, 75252 Paris cedex 05, France.
}

\begin{abstract}
We consider the seven-equation model for compressible two-phase flows and propose a large time-step numerical scheme based on a time implicit-explicit Lagrange-Projection strategy introduced in Coquel et al. [6] for Euler equations. The main objective is to get a Courant-Friedrichs-Lewy (CFL) condition driven by (slow) contact waves instead of (fast) acoustic waves.
\end{abstract}

\section{Introduction}

We are interested in the computation of compressible two-phase flows with the so-called two-fluid two-pressure or seven-equation model. It was first proposed in Baer \& Nunziato [4] and has since aroused more and more interest, see for instance Embid \& Baer [7], Stewart \& Wendroff [13], Abgrall \& Saurel [11], Gallouët, Hérard \& Seguin [8], Andrianov \& Warnecke [3], Karni et al. [9] Schwendeman, Wahle \& Kapila [12], Munkejord [10], Tokareva \& Toro [14], Ambroso, Chalons, Coquel \& Galié [1], Ambroso, Chalons \& Raviart [2], and the references therein. One of the main features of this model is that it is hyperbolic, at least in the context of subsonic flows. In particular, an interesting property is that the seven-equation model possesses seven real eigenvalues given by $\lambda_{k}^{ \pm}(\mathbf{u})=u_{k} \pm c_{k}, \lambda_{k}^{0}(\mathbf{u})=u_{k}$ and $\lambda_{I}(\mathbf{u})=u_{I}$, where $u_{k}$ denote the velocities of both phases $k=1,2, c_{k}$ the sound speeds, $u_{I}$ an interfacial velocity and $\mathbf{u}$ the vector of unknowns.

However from a numerical point of view, the seven-equation model raises some issues. The first difficulty is related to the large size of the model and as a consequence to the Riemann problem that is difficult to solve, even approximately. The second difficulty comes from the presence of nonconservative products and more precisely the fact that the model cannot be equivalently recast in full conservative form. However, the nonconservative products naturally vanish when the void fractions $\alpha_{k}$ are locally constant in space, and the model coincides in that case with two (decoupled) gas dynamics systems. This property will be used in the numerical strategy.

Numerous papers are devoted to the numerical study of two-fluid two-pressure models, see again for instance $[8],[3],[9],[12],[10],[14],[1],[2]$ and the references therein. Many of the proposed methods are based on time-explicit, exact or approximate, Godunov-type methods (Roe or Roe-like scheme, HLL or HLLC scheme...). For stability reasons, the time steps $\Delta t$ involved in such methods are subject to a usual Courant-Friedrichs-Lewy (CFL) condition that reads

$$
\max _{k, \mathbf{u}}\left(\left|\lambda_{k}^{ \pm}(\mathbf{u})\right|,\left|\lambda_{k}^{0}(\mathbf{u})\right|,\left|\lambda_{I}(\mathbf{u})\right|\right) \Delta t \leq 0.5 \Delta x
$$

where $\Delta x$ represents the space step. It is then clear that the definition of $\Delta t$ is driven by the fastest eigenvalues $\lambda_{k}^{ \pm}(\mathbf{u})$, associated with the so-called acoustic waves.

In many applications, like for instance in two-phase flows involved in nuclear reactors, the acoustic waves are not predominant physical phenomena. A CFL condition based on the most influent waves, the so-called contact waves associated with eigenvalues $\lambda_{k}^{0}(\mathbf{u})$ and $\lambda_{I}(\mathbf{u})$ would be more adapted. The idea is then to propose a timeimplicit treatment of the (fast) acoustic waves, in order to get rid of a too restrictive CFL condition, together with an explicit treatment of the (slow) contact waves in order to preserve accuracy. This was recently proposed in Coquel et al. [6] in the context of Euler equations, using a Lagrange-Projection approach. This approach is

\footnotetext{
*christophe.chalons@cea.fr

${ }^{\dagger}$ frederic. coquel@cmap.polytechnique.fr

${ }^{\ddagger}$ samuel.kokh@cea.fr

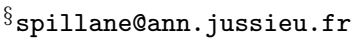


well-adapted as it naturally decouples the fast and slow waves in the Lagrange and Projection steps respectively. In this paper, we propose a first attempt to extend this approach to the seven-equation model. The idea is to operate a relevant operator splitting between the conservative and nonconservative parts of the original model, in order to make Euler systems for each phase appear. The latter parts are treated as in [6]. The nonconservative products are then discretized so as to maintain conservativity properties of the model on each partial mass, on the total momentum and total energy. Numerical results are proposed. We underline that this work is still in progress.

\section{Governing equations}

The model under consideration in this contribution reads as follows:

$$
\left\{\begin{array}{l}
\partial_{t} \alpha_{k}+u_{I} \partial_{x} \alpha_{k}=0, \quad t>0, \quad x \in \mathbb{R} \\
\partial_{t} \alpha_{k} \varrho_{k}+\partial_{x} \alpha_{k} \varrho_{k} u_{k}=0 \\
\partial_{t} \alpha_{k} \varrho_{k} u_{k}+\partial_{x} \alpha_{k}\left(\varrho_{k} u_{k}^{2}+p_{k}\right)-p_{I} \partial_{x} \alpha_{k}=0 \\
\partial_{t} \alpha_{k} \varrho_{k} e_{k}+\partial_{x} \alpha_{k}\left(\varrho_{k} e_{k}+p_{k}\right) u_{k}-p_{I} u_{I} \partial_{x} \alpha_{k}=0
\end{array}\right.
$$

with $k=1,2$. Here, $\alpha_{k}, \varrho_{k}, u_{k}, e_{k}$ and $p_{k}$ denote the volume fraction, density, velocity, specific total energy and pressure of the phase $k=1,2$. The two phases are assumed to be non-miscible that is $\alpha_{1}+\alpha_{2}=1$. The structure of (1) is the one of two gas dynamics systems for each phase, coupled with a transport equation on the void fraction $\alpha_{k}$ at speed $u_{I}$. We note that nonconservative products involving the interfacial pressure $p_{I}$ and velocity $u_{I}$ (to be precised later on) and the space derivative of the void fractions $\alpha_{k}$ are present in the momentum and energy equations. These terms act as coupling terms in the evolution of the two phases. Source terms like external forces, pressure and velocity relaxations, dissipation, heat conduction, phase changes and heat exchanges between the two phases are not taken into account.

Each phase is provided with an equation of state $p_{k}=p_{k}\left(\varrho_{k}, \varepsilon_{k}\right)$, where $\varepsilon_{k}=e_{k}-u_{k}^{2} / 2$ is the specific internal energy. So far as the definitions of $u_{I}$ and $p_{I}$ are concerned, we follow [8] and first observe that the characteristic speeds of (1) are always real and given by $u_{I}, u_{k}, u_{k} \pm c_{k}, k=1,2$, where $c_{k}$ denotes the speed of sound in phase $k$. System (1) turns out to be only weakly hyperbolic since there are not enough eigenvectors to span the entire space when $u_{I}=u_{k} \pm c_{k}$ for some index $k$ (resonance occurs). When (1) is hyperbolic, one can easily check that similarly to the classical gas dynamics equations, the characteristic fields associated with the eigenvalues $u_{k} \pm c_{k}$ are nonlinear while the one associated with $u_{k}$ is linearly degenerate. Regarding the characteristic field associated with $u_{I}$, it is generally required to be linearly degenerate in practice. This property holds as soon as

$$
u_{I}=\beta u_{1}+(1-\beta) u_{2}, \quad \beta=\frac{\chi \alpha_{1} \varrho_{1}}{\chi \alpha_{1} \varrho_{1}+(1-\chi) \alpha_{2} \varrho_{2}}
$$

where $\chi \in[0,1]$ is a constant (we refer to [8] for the details), which gives a natural definition for the interfacial velocity $u_{I}$. The usual choices for $\chi$ are $0,1 / 2$ and 1 . Regarding the interfacial pressure $p_{I}$, we set $p_{I}=$ $\mu p_{1}+(1-\mu) p_{2}, \mu \in[0,1]$. The choice of the coefficient $\mu$ is not detailed here (see again [8]) but is related to the ability to provide the system with an entropy balance equation. Indeed, it can be proved that for a specific choice of $\mu$, smooth solutions of (1) verify the conservation law $\partial_{t} \eta+\partial_{x} q=0$, where $(\eta, q)$ plays the role of a mathematical entropy pair.

\section{A natural operator splitting}

The starting point is to propose an equivalent form of (1) where the space derivatives of $\alpha_{k} p_{k}$ and $\alpha_{k} p_{k} u_{k}$ are decomposed using a chain rule:

$$
\left\{\begin{array}{l}
\partial_{t} \alpha_{k}+u_{I} \partial_{x} \alpha_{k}=0 \\
\partial_{t} \alpha_{k} \varrho_{k}+\partial_{x} \alpha_{k} \varrho_{k} u_{k}=0 \\
\partial_{t} \alpha_{k} \varrho_{k} u_{k}+\partial_{x} \alpha_{k} \varrho_{k} u_{k}^{2}+\alpha_{k} \partial_{x} p_{k}+\left(p_{k}-p_{I}\right) \partial_{x} \alpha_{k}=0 \\
\partial_{t} \alpha_{k} \varrho_{k} e_{k}+\partial_{x} \alpha_{k} \varrho_{k} e_{k} u_{k}+\alpha_{k} \partial_{x} p_{k} u_{k}+\left(p_{k} u_{k}-p_{I} u_{I}\right) \partial_{x} \alpha_{k}=0
\end{array}\right.
$$

We then suggest to split (3) into two independent and quasi-classical gas dynamics equations (their Lagrangian forms will be seen to be classical), namely

$$
\left\{\begin{array}{l}
\partial_{t} \alpha_{k}=0 \\
\partial_{t} \alpha_{k} \varrho_{k}+\partial_{x} \alpha_{k} \varrho_{k} u_{k}=0 \\
\partial_{t} \alpha_{k} \varrho_{k} u_{k}+\partial_{x} \alpha_{k} \varrho_{k} u_{k}^{2}+\alpha_{k} \partial_{x} p_{k}=0 \\
\partial_{t} \alpha_{k} \varrho_{k} e_{k}+\partial_{x} \alpha_{k} \varrho_{k} e_{k} u_{k}+\alpha_{k} \partial_{x} p_{k} u_{k}=0
\end{array}\right.
$$


and into the following genuinely nonconservative system:

$$
\left\{\begin{array}{l}
\partial_{t} \alpha_{k}+u_{I} \partial_{x} \alpha_{k}=0 \\
\partial_{t} \alpha_{k} \varrho_{k}=0 \\
\partial_{t} \alpha_{k} \varrho_{k} u_{k}+\left(p_{k}-p_{I}\right) \partial_{x} \alpha_{k}=0 \\
\partial_{t} \alpha_{k} \varrho_{k} e_{k}+\left(p_{k} u_{k}-p_{I} u_{I}\right) \partial_{x} \alpha_{k}=0
\end{array}\right.
$$

This transformation aims at proposing in the next section an implicit-explicit Lagrange-Projection scheme similar to [6], and at treating separately the nonconservative products. Note from now on that the overall algorithm will be conservative on the partial mass $\alpha_{k} \varrho_{k}$, total momentum $\alpha_{1} \varrho_{1} u_{1}+\alpha_{2} \varrho_{2} u_{2}$ and on the total energy $\alpha_{1} \varrho_{1} e_{1}+\alpha_{2} \varrho_{2} e_{2}$, as it is expected from the original form (1) of the model.

\section{Numerical approximation}

This section is devoted to the discretization of (1), using (4) and (5). Let us introduce a time step $\Delta t>0$ and a space step $\Delta x>0$ that we assume to be constant for simplicity. We set $\lambda=\Delta t / \Delta x$ and define the mesh interfaces $x_{j+1 / 2}=j \Delta x$ for $j \in \mathbb{Z}$, and the intermediate times $t^{n}=n \Delta t$ for $n \in \mathbb{N}$. In the sequel, $\mathbf{u}_{j}^{n}=\left(\alpha_{1}, \mathbf{u}_{1}, \mathbf{u}_{2}\right)_{j}^{n}$ where $\left(\mathbf{u}_{k}\right)_{j}^{n}=\left(\alpha_{k} \varrho_{k}, \alpha_{k} \varrho_{k} u_{k}, \alpha_{k} \varrho_{k} e_{k}\right)_{j}^{n}$ denotes the approximate value of the unknowns at time $t^{n}$ and on the cell $\left.\mathcal{C}_{j}=\right] x_{j-1 / 2}, x_{j+1 / 2}[$.

Implicit-explicit discretization of (4). We first recall that (4) is made of two independent quasi-classical gas dynamics systems, whose eigenvalues are given by $u_{k} \pm c_{k}, u_{k}$ and 0 . As already stated, our aim is to propose an implicit treatment of the fast waves $u_{k} \pm c_{k}$, and an explicit treatment of $u_{k}$. With this in mind, we follow [6] and adopt a Lagrange-Projection scheme, coupled with a pressure relaxation strategy that is well adapted to this purpose. A Lagrange-Projection splitting strategy applied to (4) amounts to introducing the Lagrangian system

$$
\left\{\begin{array} { l } 
{ \partial _ { t } \alpha _ { k } = 0 , } \\
{ \partial _ { t } \alpha _ { k } \varrho _ { k } + \alpha _ { k } \varrho _ { k } \partial _ { x } u _ { k } = 0 , } \\
{ \partial _ { t } \alpha _ { k } \varrho _ { k } u _ { k } + \alpha _ { k } \varrho _ { k } u _ { k } \partial _ { x } u _ { k } + \alpha _ { k } \partial _ { x } p _ { k } = 0 , } \\
{ \partial _ { t } \alpha _ { k } \varrho _ { k } e _ { k } + \alpha _ { k } \varrho _ { k } e _ { k } \partial _ { x } u _ { k } + \alpha _ { k } \partial _ { x } p _ { k } u _ { k } = 0 , }
\end{array} \quad \text { or equivalently } \quad \left\{\begin{array}{l}
\partial_{t} \alpha_{k}=0 \\
\partial_{t} \tau_{k}-\tau_{k} \partial_{x} u_{k}=0 \\
\partial_{t} u_{k}+\tau_{k} \partial_{x} p_{k}=0 \\
\partial_{t} e_{k}+\tau_{k} \partial_{x} p_{k} u_{k}=0
\end{array}\right.\right.
$$

with $\tau_{k}=1 / \rho_{k}$, and the transport (or projection) system

$$
\left\{\begin{array}{l}
\partial_{t} \alpha_{k}=0 \\
\partial_{t} \alpha_{k} \varrho_{k}+u_{k} \partial_{x} \alpha_{k} \varrho_{k}=0 \\
\partial_{t} \alpha_{k} \varrho_{k} u_{k}+u_{k} \partial_{x} \alpha_{k} \varrho_{k} u_{k}=0 \\
\partial_{t} \alpha_{k} \varrho_{k} e_{k}+u_{k} \partial_{x} \alpha_{k} \varrho_{k} e_{k}=0
\end{array}\right.
$$

We note that (6) coincides with two classical gas dynamics systems written in Lagrangian coordinates, the eigenvalues of which are given by $\pm c_{k}$ and 0 . This system is treated using a pressure relaxation approach that consists in introducing a linearized pressure $\pi_{k}$ (see for instance [5] and especially the references therein), such that $\left(\pi_{k}\right)_{j}^{n}=\left(p_{k}\right)_{j}^{n}$, and in solving the partial differential system

$$
\left\{\begin{array} { l } 
{ \partial _ { t } \alpha _ { k } = 0 , } \\
{ \partial _ { t } \tau _ { k } - \tau _ { k } \partial _ { x } u _ { k } = 0 , } \\
{ \partial _ { t } u _ { k } + \tau _ { k } \partial _ { x } \pi _ { k } = 0 , } \\
{ \partial _ { t } \pi _ { k } + a _ { k } ^ { 2 } \tau _ { k } \partial _ { x } u _ { k } = 0 , } \\
{ \partial _ { t } e _ { k } + \tau _ { k } \partial _ { x } \pi _ { k } u _ { k } = 0 , }
\end{array} \quad \text { or equivalently } \left\{\begin{array}{l}
\partial_{t} \alpha_{k}=0 \\
\partial_{t} I_{k}=0 \\
\partial_{t} w_{k}^{+}+a_{k} \tau_{k} \partial_{x} w_{k}^{+}=0 \\
\partial_{t} w_{k}^{-}-a_{k} \tau_{k} \partial_{x} w_{k}^{-}=0 \\
\partial_{t} e_{k}+\tau_{k} \partial_{x} \pi_{k} u_{k}=0
\end{array}\right.\right.
$$

where $w_{k}^{ \pm}=\pi_{k} \pm a_{k} u_{k}, I_{k}=\pi_{k}+a_{k}^{2} \tau_{k}$, and $a_{k}$ is a constant satisfying the subcharacteristic condition $a_{k}>\rho_{k} c_{k}$. A natural time-implicit discretization of (8) is

$$
\left\{\begin{array}{l}
\left(\alpha_{k}\right)_{j}^{n+1=}=\left(\alpha_{k}\right)_{j}^{n}, \\
\left(I_{k}\right)_{j}^{n+1=}=\left(I_{k}\right)_{j}^{n}, \\
\left(w_{k}^{+}\right)_{j}^{n+1=}=\left(w_{k}^{+}\right)_{j}^{n}-\lambda\left(\tau_{k}\right)_{j}^{n} a_{k}\left(\left(w_{k}^{+}\right)_{j}^{n+1=}-\left(w_{k}^{+}\right)_{j-1}^{n+1=}\right), \\
\left(w_{k}^{-}\right)_{j}^{n+1=}=\left(w_{k}^{-}\right)_{j}^{n}+\lambda\left(\tau_{k}\right)_{j}^{n} a_{k}\left(\left(w_{k}^{+}\right)_{j+1}^{n+1=}-\left(w_{k}^{+}\right)_{j}^{n+1=}\right), \\
\left(e_{k}\right)_{j}^{n+1=}=\left(e_{k}\right)_{j}^{n}-\lambda\left(\tau_{k}\right)_{j}^{n}\left(\left(\pi_{k} u_{k}\right)_{j+1 / 2}^{n+1=}-\left(\pi_{k} u_{k}\right)_{j-1 / 2}^{n+1=}\right),
\end{array}\right.
$$

with $\left(\pi_{k} u_{k}\right)_{j+1 / 2}^{n+1=}=\left(\pi_{k}\right)_{j+1 / 2}^{n+1=}\left(u_{k}\right)_{j+1 / 2}^{n+1=}$ and

$$
\left(\pi_{k}\right)_{j+1 / 2}^{n+1=}=\frac{1}{2}\left(\left(w_{k}^{+}\right)_{j}^{n+1=}+\left(w_{k}^{-}\right)_{j}^{n+1=}\right), \quad\left(u_{k}\right)_{j+1 / 2}^{n+1=}=\frac{1}{2 a_{k}}\left(\left(w_{k}^{+}\right)_{j}^{n+1=}-\left(w_{k}^{-}\right)_{j}^{n+1=}\right) .
$$


The updated values of $u_{k}, \tau_{k}$ and $\rho_{k}$ are recovered from the formulas $u_{k}=\left(w_{k}^{+}-w_{k}^{-}\right) / 2 a_{k}, \pi_{k}=\left(w_{k}^{+}+w_{k}^{-}\right) / 2$, $\tau_{k}=\left(I_{k}-\pi_{k}\right) / a_{k}^{2}$ and $\rho_{k}=1 / \tau_{k}$. The computation of $\left(w_{k}^{ \pm}\right)_{j}^{n+1=}$ is cheap and amounts to solving a tridiagonal system of linear equations, while the time-implicit definition of $\left(e_{k}\right)_{j}^{n+1=}$ explicitly follows.

Then, the transport equations involved in (7) are associated with the following classical time-explicit update formula

$$
\begin{aligned}
\left(\mathbf{u}_{k}\right)_{j}^{n+1-}=\left(\mathbf{u}_{k}\right)_{j}^{n+1=}+\lambda & ( \\
& \max \left(\left(u_{k}\right)_{j-1 / 2}^{n+1=}, 0\right)\left(\mathbf{u}_{k}\right)_{j-1}^{n+1=}-\min \left(\left(u_{k}\right)_{j+1 / 2}^{n+1=}, 0\right)\left(\mathbf{u}_{k}\right)_{j+1}^{n+1=} \\
& + \\
& {\left[\min \left(\left(u_{k}\right)_{j+1 / 2}^{n+1=}, 0\right)-\max \left(\left(u_{k}\right)_{j-1 / 2}^{n+1=}, 0\right)\right]\left(\mathbf{u}_{k}\right)_{j}^{n+1=} } \\
& ),
\end{aligned}
$$

and of course $\left(\alpha_{k}\right)_{j}^{n+1-}=\left(\alpha_{k}\right)_{j}^{n+1=}$.

Discretization of (5). Our objective is to propose a consistent approximation of (5) such that the overall algorithm is conservative for each partial mass, for the total momentum and for the total energy, as already motivated. First of all and similarly to (10), the transport equation associated with $\alpha_{k}$ is treated as follows:

$$
\begin{aligned}
\left(\alpha_{k}\right)_{j}^{n+1}=\left(\alpha_{k}\right)_{j}^{n+1=}+\lambda & ( \\
& \max \left(\left(u_{I}\right)_{j-1 / 2}^{n+1=}, 0\right)\left(\alpha_{k}\right)_{j-1}^{n+1=}-\min \left(\left(u_{I}\right)_{j+1 / 2}^{n+1=}, 0\right)\left(\alpha_{k}\right)_{j+1}^{n+1=} \\
& +\ldots\left(\min \left(\left(u_{I}\right)_{j+1 / 2}^{n+1=}, 0\right)-\max \left(\left(u_{I}\right)_{j-1 / 2}^{n+1=}, 0\right)\right]\left(\alpha_{k}\right)_{j}^{n+1=}
\end{aligned}
$$

where $\left(u_{I}\right)_{j+1 / 2}^{n+1=}=\beta_{j+1 / 2}^{n+1=}\left(u_{1}\right)_{j+1 / 2}^{n+1=}+\left(1-\beta_{j+1 / 2}^{n+1=}\right)\left(u_{2}\right)_{j+1 / 2}^{n+1=}$ and for instance $\beta_{j+1 / 2}^{n+1=}=\frac{1}{2}\left(\beta_{j}^{n+1=}+\beta_{j+1}^{n+1=}\right)$. We set $\left(\alpha_{k} \rho_{k}\right)_{j}^{n+1}=\left(\alpha_{k} \rho_{k}\right)_{j}^{n+1-}$ for the partial mass, so that only the treatments of the momentum and total energy of each phase are now left. We propose

$$
\begin{gathered}
\frac{\left(\alpha_{k} \rho_{k} u_{k}\right)_{j}^{n+1}-\left(\alpha_{k} \rho_{k} u_{k}\right)_{j}^{n+1-}}{\Delta t}+\left(\left(\overline{p_{k}}\right)_{j}-\left(\overline{p_{I}}\right)_{j}\right) \frac{\left(\alpha_{k}\right)_{j+1 / 2}^{n}-\left(\alpha_{k}\right)_{j-1 / 2}^{n}}{\Delta x}=0 \\
\frac{\left(\alpha_{k} \rho_{k} e_{k}\right)_{j}^{n+1}-\left(\alpha_{k} \rho_{k} e_{k}\right)_{j}^{n+1-}}{\Delta t}+\left(\left(\overline{p_{k} u_{k}}\right)_{j}-\left(\overline{p_{I} u_{I}}\right)_{j}\right) \frac{\left(\alpha_{k}\right)_{j+1 / 2}^{n}-\left(\alpha_{k}\right)_{j-1 / 2}^{n}}{\Delta x}=0 .
\end{gathered}
$$

In order to get the expected overall conservativity properties, we pay a particular attention to the definitions of $\left(\overline{p_{k}}\right)_{j},\left(\overline{p_{I}}\right)_{j},\left(\overline{p_{k} u_{k}}\right)_{j}$ and $\left(\overline{p_{I} u_{I}}\right)_{j}$. For any consistent definition of the flux $\left(\alpha_{k}\right)_{j+1 / 2}^{n}$, we set with $\kappa_{j} \in[0,1]$

$$
\left\{\begin{array}{l}
\left(\alpha_{k}\right)_{j}^{n}=\kappa_{j}\left(\alpha_{k}\right)_{j+1 / 2}^{n}+\left(1-\kappa_{j}\right)\left(\alpha_{k}\right)_{j-1 / 2}^{n} \\
\left(\overline{p_{k}}\right)_{j}=\left(1-\kappa_{j}\right)\left(\pi_{k}\right)_{j+1 / 2}^{n+1=}+\kappa_{j}\left(\pi_{k}\right)_{j-1 / 2}^{n+1=} \\
\left(\overline{p_{k} u_{k}}\right)_{j}=\left(1-\kappa_{j}\right)\left(\pi_{k} u_{k}\right)_{j+1 / 2}^{n+1=}+\kappa_{j}\left(\pi_{k} u_{k}\right)_{j-1 / 2}^{n+1=}
\end{array}\right.
$$

and

$$
\left\{\begin{array}{l}
\left(\overline{p_{I}}\right)_{j}=\mu_{j+1 / 2}^{n+1=}\left(\overline{p_{1}}\right)_{j}+\left(1-\mu_{j+1 / 2}^{n+1=}\right)\left(\overline{p_{2}}\right)_{j}, \quad \text { with } \mu_{j+1 / 2}^{n+1=}=\frac{1}{2}\left(\mu_{j}^{n+1=}+\mu_{j+1}^{n+1=}\right) \\
\left(\overline{u_{I}}\right)_{j}=\beta_{j+1 / 2}^{n+1=}\left(\overline{u_{1}}\right)_{j}+\left(1-\beta_{j+1 / 2}^{n+1=}\right)\left(\overline{u_{2}}\right)_{j}, \quad \text { with }\left(\overline{u_{k}}\right)_{j}=\left(\overline{p_{k} u_{k}}\right)_{j} /\left(\overline{p_{k}}\right)_{j}, \\
\left(\overline{p_{I} u_{I}}\right)_{j}=\left(\overline{p_{I}}\right)_{j}\left(\overline{u_{I}}\right)_{j} .
\end{array}\right.
$$

We choosed in practice $\left(\alpha_{k}\right)_{j+1 / 2}^{n}=\left(\alpha_{k}\right)_{j}^{n}$ or equivalently $\kappa_{j}=1$.

With such definitions, it can be proved that under a suitable CFL condition based on the velocities $u_{k}$ and $u_{I}$ only, and not on the acoustic waves $u_{k} \pm c_{k}$, the void fractions $\left(\alpha_{k}\right)_{j}^{n+1}$ belong to $(0,1)$ if $\left(\alpha_{k}\right)_{j}^{n}$ do. We can also prove that under the same restriction on the time step $\left(\rho_{k}\right)_{j}^{n+1}$ is positive, as well as $\left(\varepsilon_{k}\right)_{j}^{n+1-}$ and $\left(p_{k}\right)_{j}^{n+1-}$. Unfortunately, the positivity of $\left(\varepsilon_{k}\right)_{j}^{n+1}$ and $\left(p_{k}\right)_{j}^{n+1}$ is not proved at the moment.

\section{$5 \quad$ Numerical experiments}

For the sake of illustration, we present in this section the results given by our algorithm on three Riemann problems. They are all taken from [2] and are fully described therein. Space and time orders of accuracy are one. The first one (top left) corresponds to an isolated contact discontinuity propagating with a positive velocity, while 

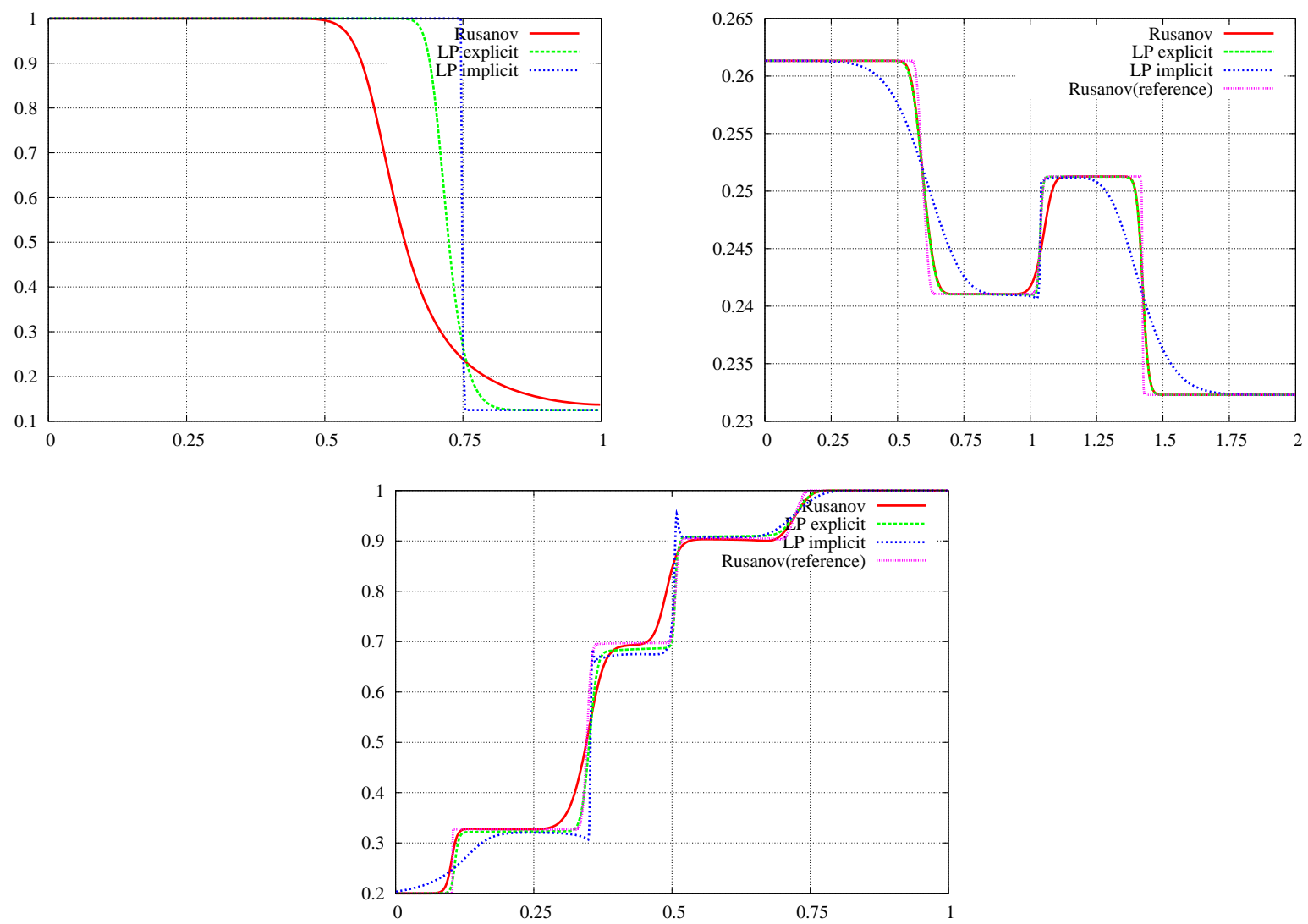

Figure 1: Comparison of several schemes with a reference solution (density profile)

\begin{tabular}{|c|c|c|c|}
\hline & Test 1 & Test 2 & Test 3 \\
\hline Rusanov & 4231 & 550 & 2630 \\
LP explicit & 4297 & 551 & 2631 \\
LP implicit & 63 & 41 & 151 \\
\hline
\end{tabular}

Table 1: Number of time-iterations for each test case

the second one (top right) and the third one (bottom) involve several distinct waves. The scheme we propose here is denoted LP implicit and is compared with its explicit version (which amounts to replacing (9) by its time-explicit version) and the well-known Rusanov scheme (see [8]). We observe that our approach is clearly less diffusive around the contact discontinuities since the CFL condition is well-adapted to the corresponding speed of propagation, but more diffusive around the acoustic waves since it is implicit. Table 1 gives for each test case the number of iterations needed to perform the computations. As expected, the gain is important when using the proposed implicit-explicit algorithm and the corresponding CFL restriction based on the material waves (instead of the acoustic waves as for the explicit scheme). A careful evaluation of the CPU cost necessitates an additional programming effort that has not been implemented yet.

\section{References}

[1] A. Ambroso, C. Chalons, F. Coquel, T. Galié. Relaxation and numerical approximation of a two fluid two pressure diphasic model. M2AN, vol. 43, pp. 1063-1097, (2009).

[2] A. Ambroso, C. Chalons and P.-A. Raviart. A Godunov-type method for the seven-equation model of compressible two-phase flow. LJLL report number R10020, http://www.ljll.math.upmc.fr/publications/2010/R10020.php, (2010).

[3] N. Andrianov and G. Warnecke. The Riemann problem for the Baer-Nunziato two-phase flow model. Journal of Computational Physics, vol. 195, pp. 434-464, (2004). 
[4] M.R. Baer and J.W. Nunziato, A two phase mixture theory for the deflagration to detonation transition in reactive granular materials. Int. J. Mult. Flows, vol. 12(6), pp. 861-889, (1986).

[5] C. Chalons and J.-F. Coulombel, Relaxation approximation of the Euler equations. J. Math. Anal. Appl., vol. 348(2), pp. 872-893, (2008).

[6] F. Coquel, Q.-L. Nguyen, M. Postel and Q.-H. Tran, Entropy-satisfying relaxation method with large time-steps for Euler IBVPs. Math. Comp, vol. 79, pp. 1493-1533, (2010).

[7] P. Embid and M. Baer, Mathematical analysis of a two-phase continuum mixture theory, Contin. Mech. Thermodyn. vol. 4(4), pp. 279-312, (1992).

[8] T. Gallouët, J.-M. Hérard and N. Seguin. Numerical modeling of two-phase flows using the two-fluid twopressure approach. M3AS, vol. 14(5), pp. 663-700, (2004).

[9] S. Karni, E. Kirr, A. Kurganov and G. Petrova, Compressible two-phase flows by central and upwind schemes, M2AN, vol. 38(3), pp. 477-493, (2004).

[10] S.T. Munkejord, Comparison of Roe-type methods for solving the two-fluid model with and without pressure relaxation, Computers and Fluids, vol. 36, pp. 1061-1080, (2007).

[11] R. Saurel and R. Abgrall, A multiphase Godunov method for compressible multifluid and multiphase flows, J. Comput. Phys., vol. 150, pp. 425-467, (1999).

[12] D.-W. Schwendeman, C.-W. Wahle and A.-K. Kapila. The Riemann problem and a high-resolution Godunov method for a model of compressible two-phase flow. J.C.P.(2006).

[13] B. Stewart and B. Wendroff, Two-phase flow : models and methods, J. Comp. Phys. (1984).

[14] S.-A. Tokareva and E.-F. Toro, HLLC-type Riemann solver for the Baer-Nunziato equations of compressible two-phase flow, J. Comput. Phys., to appear, (2010). 